\title{
螺旋通道内流体流动与传热特性研究进展
}

林清宇 ${ }^{1,2^{*}}$, 刘鹏辉 ${ }^{1,2}$, 冯振飞 ${ }^{1,2}$, 朱礼 ${ }^{1,2}$

1. 广西大学广西石化资源加工及过程强化技术重点实验室, 南宁 530004;

2. 广西大学化学化工学院, 南宁 530004

* 联系人, E-mail: linqy121@gxu.edu.cn

2016-10-12 收稿, 2017-03-13 修回, 2017-03-14 接受, 2017-05-12 网络版发表

广西自然科学基金(2014GXNSFBA118051)、广西石化资源加工及过程强化技术重点实验室主任基金(2016Z013, 2015Z012)和广西大学科研 基金(XJZ130359)资助

摘要螺旋通道是提高流体传热及传质效率的重要结构，以其节省空间及易于加工的特点被广泛应用。而通道 内流体的流动和传热特性作为评价螺旋通道的重要性质，对其实际应用具有重要的指导意义，成为近年来的研究 热点. 而研究重点主要集中在螺旋通道的结构参数和流动工质两个方面, 本文对螺旋通道内流体流动与传热特性 研究进行综述, 总结了螺旋通道结构及流动工质对其性能的影响规律; 具体分析了螺旋通道直径、管径、螺距、 截面形状等结构参数以及流动工质种类、浓度等物性参数对其传热系数和流动阻力的影响; 对比了层流及湍流状 态下实验和数值模拟结论; 为螺旋通道结构优化及工质选取提供了参考, 并且展望了螺旋通道内流体流动及传热 特性研究的发展趋势.

关键词螺旋通道, 结构, 工质, 流动特性, 传热特性

螺旋通道以其结构紧凑、易于加工及传热效率高 等优点被广泛应用于发电、制冷、化学反应、热量回 收、食品加工及物料混合等领域. 与普通直管不同, 螺旋通道能够产生垂直于轴向流动方向的二次流, 可以增强流体的扰动, 进而减薄边界层厚度. Goering 等人 ${ }^{[1]}$ 已经证实螺旋通道中产生的二次流动占平均 流体流动的 16\% 20\%, 而二次流动产生的原因正是 螺旋管道曲率产生的离心力对流体的作用. 如图 1所 示, 螺旋通道的几何及设计参数包括了螺旋半径 $R$ 、管 径 $D$ 及螺距 $P$. 吴双应等人 ${ }^{[2] 、 K o}$ 等人 $^{[3,4] 、 C i o n c o l i n i ~}$ 等人 ${ }^{[5]}$ 及 Chen等人 ${ }^{[6]}$ 探究了上述几何参数以及螺旋 通道曲率、扭率等对其流动和传热特性的影响, 并从 阻力系数、努塞尔数、熵产等方面对其性能进行了评 价, 为螺旋通道的结构优化提供了一定参考.

而近年来, 纳米流体以其自身的热物理性质、潜

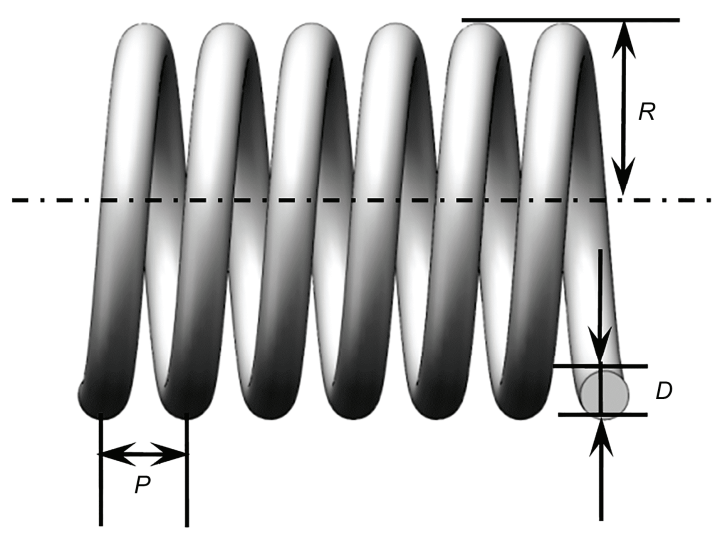

图 1 螺旋通道的几何和设计参数

Figure 1 Geometry and design parameters of helically coiled tube

在的优点及广泛的应用吸引了越来越多的学者对其 进行研究. 纳米流体是在基液中添加纳米级颗粒物 
和分散剂而制成的, 其中纳米颗粒包括了金属单质 (例如, $\mathrm{Cu}, \mathrm{Ag}$ 等)、金属氧化物(例如, $\mathrm{Al}_{2} \mathrm{O}_{3}, \mathrm{TiO}_{2}$ 等) 及碳的同素异形体(例如, 金刚石、单层及多层碳纳 米管等). 布朗运动作为纳米流体的一个显著特性, 能够显著提升其传热效果. Sundar等人 ${ }^{[7]}$ 及 $\mathrm{Li}^{\text {等人 }}{ }^{[8]}$ 通过研究指出, 纳米颗粒的加人对于基液传热系数 提升相当明显, 但纳米流体与基液相比也具有更高 的流动阻力. 所以, 在探究不同流动工质传热特性的 同时, 也应该对其流动特性进行分析, 这样才能更加 合理地指导换热工质的选取及使用.

改变螺旋通道结构及流动工质的最终目的都是 为了提高其传热性能, 并且尽量减少流动阻力. 虽然 现今已有很多关于螺旋通道内流体流动及传热特性 方面的综述, 但是综合两者进行总结的并不多, 并且 对于分析其结构及工质, 特别是几何参数及纳米流 体对其流动及传热特性影响的综述还很少.

本文将从螺旋通道结构及流动工质两个方面出 发, 特别是其几何参数和纳米流体工质, 对近几年来 螺旋通道内流体流动及传热特性的实验和数值模拟 研究进行综述, 希望对以后的研究者有一定帮助.

\section{1 螺旋通道结构}

相比于直通道, 螺旋通道自身结构的曲率和扭 率使得流经介质的流动状态变得相对复杂, 离心力 和向心力的作用使得流体在流动过程中偏向壁面而 形成迪恩浴, 流动场和速度场均发生改变. 相应地, 其流动状态的判定标准也有所不同, 直通道中, 当雷 诺数 $R e$ 小于 2300 时称作层流, 2300 4000时为过渡区, 超过 4000 则为湍流 ${ }^{[9]}$; 而在螺旋通道中, 层流、湍流 临界雷诺数随着螺旋通道结构参数的改变而变化, 具体计算公式为

$$
\begin{gathered}
R e_{\mathrm{c}}=2 \times 10^{4} \cdot \delta^{0.32}, \\
\delta=\frac{D}{2 R},
\end{gathered}
$$

当 $\delta^{-1} \neq 860$, 雷诺数 $R e$ 小于 $R e_{\mathrm{c}}$ 时, 流体的流动状态 为层流, 大于 $R e_{\mathrm{c}}$ 则为湍流 ${ }^{[10]}$, 本文就将以此对螺旋 通道内流体的流动和传热特性进行概括分析.

\section{1 层流状态下的流动特性}

(i ) 实验研究. 螺旋通道自身的几何参数会在 一定程度上影响流体的流动特性, 近年来, 越来越多 的学者通过实验对其进行了探究. Ghobadi等人 ${ }^{[11]}$ 就
研究了小尺寸螺旋管的压降, 发现低雷诺数下, 曲率 越大, 压降越大. 说明在流体流动的过程中, 扰动的 增强提高了压降. 张丽等人 ${ }^{[12]}$ 通过实验, 研究了矩 形截面螺旋通道内流体的流动特性, 结果发现, 由于 离心力和扭转力的作用, 其轴向速度最大值偏向外 壁, 流动截面上会产生两个二次涡; 随着矩形截面高 宽比的增大, 轴向速度又偏向上半截面, 而两个二次 浴分别移向两侧; 随着矩形截面高宽比的减小, 二次 流动增强, 阻力系数也增大.

这就表明, 曲率在螺旋管道的结构优化中十分 重要, 增加曲率可以提高其流动阻力; 而矩形螺旋通 道上、下壁面处的扰动已经相当剧烈, 截面中心处较 弱, 由此, 可以通过内置浴发生器或者扰流元件的方 法来提高整体的扰动效果.

马源等人 ${ }^{[13]}$ 发现, 随着螺旋通道螺旋角的上升, 横截面的二次流高速区外移, 使得外侧速度增加. Liu等人 ${ }^{[14,15]}$ 通过可视化实验, 对水平放置矩形截面 螺旋通道内弹状流的流动特性进行了实验研究. 结 果表明: 其流动特性会随着螺旋角位置的改变而变 化. 重力和离心力的相对大小决定着内、外壁面液膜 的厚度, 在螺旋上升过程中, 液膜下降速度逐渐减 小, 在螺旋下降段, 液膜速度明显增加, 气弹往螺旋 通道上侧运动的过程中, 流速逐渐减小; 液塞速度随 着液体表面流速的增加而增大; 壁面两侧液膜降落 速度相同, 并随着气体流速的增加而增大; 液体流速 增加时, 液膜厚度也随之增大.

由上述研究可知, 螺旋角度上升而引起的螺旋 通道内流体高速区外移, 靠近壁面附近的流体流动 状态发生改变, 流体流动阻力也会随着高速区外移 的程度而发生变化, 这就为螺旋通道流动特性优化 提供依据; 而可视化的研究需要结合螺旋通道局部 及整体流动和传热的变化进行分析, 以此来评价不 同流型与流动和传热特性的关系, 才能更加具体地 为结构优化提供参考.

(ii) 模拟研究. 除了张丽等人 ${ }^{[12]}$ 对矩形截面 螺旋通道流动特性进行数值模拟, 并得到和实验测 量值相近的结果外, Mohammed等人 ${ }^{[16]}$ 模拟了不同螺 距对其压降的影响, 发现改变螺距对压降并无明显 改变; 而随着螺旋半径的减小和螺旋管内径的增加, 压降增大. 吴双应等人 ${ }^{[17]}$ 研究了无量纲螺距和曲率 对环形螺旋通道流动特性的影响. 结果发现, 其流动 阻力随着曲率的增加而增加, 但受无量纲螺距的影 
响很小. 由此可知, 螺旋通道曲率是影响其流动特性 的重要因素, 可以通过改变曲率对其流动特性进行 优化.

随后, Nobari等人 ${ }^{[18]}$ 探究了理查森数R $i$ 对螺旋通 道混合对流的影响. 结果发现, 在浮力的作用下, 通 道内会产生一对不对称浴, 使得流动变得更加复杂, 从而达到强化传热的效果.

上述研究均从具体的螺旋通道结构参数出发, 分析它们在层流状态下对其流动特性的影响, 相对 于湍流而言, 层流状态下流体紊乱程度较低, 通过改 变螺旋通道曲率可以有效改变其流动特性.

\section{2 湍流状态下的流动特性}

( i ) 实验研究. 相比于层流状态, 湍流状态下 的流动紊乱程度较高, 研究主要集中在螺旋通道中 的扰流装置上, 通过二次流发生装置来进一步提高 流体的扰动.

由张丽等人 ${ }^{[12]}$ 的实验结果可知, 矩形截面螺旋 通道内靠近壁面处的扰动比中间截面强烈, 一些学 者就通过加人浴发生器的方法来增强截面中间流体 的扰动效果. 随后, 张丽等人 ${ }^{[19]}$ 就对安装三角翼形 浴发生器的矩形截面螺旋通道进行了研究. 结果表 明: 湍流状态下, 内壁处会出现两个新的二次浴, 浴 量最大值为光管的 2.0 2.8倍, 有效地增强了扰动. 而螺旋通道中的两相流在离心力的作用下要比直管中 的情况更加复杂, 所以, Saffari等人 ${ }^{[20]}$ 通过实验探究 了螺旋通道气液两相的压降及流阻. 结果表明: 随着 雷诺数及空隙率的增加, 流阻减小; 随着曲率的减小, 流阻增大; 随着雷诺数的增加, 注人气泡的直径减小.

(ii) 模拟研究. 通过数值模拟对结构进行优 化, 然后选取合适的结构参数对其进行实验研究, 可 以节约时间, 提高实验效率. Xia等人 ${ }^{[21]}$ 数值模拟了 矩形截面螺旋通道内气液两相流的压降, 结果表明, 螺旋通道内气液两相流单位螺旋管道长度的摩擦压 降及摩擦阻力的梯度均随着曲率的增加而增大; 而 随着扭率的增加, 摩擦阻力及压降也会增大. 陈瑞杰 等人 ${ }^{[22]}$ 在矩形截面螺旋通道内加人肋片, 使得矩形 截面中心处产生附加二次流, 流动阻力系数较光管 增加了 $0.3 \%$ 3.3\%. 李雅侠等人 ${ }^{[23]}$ 研究了不同形状 和不同布置方式的翼型浴发生器对半圆形截面螺旋 通道的流动特性的影响, 结果表明, 其流动阻力系数 与光管流动阻力之比 $f / f_{0}$ 在 $1.105 \sim 1.188$ 之间. 也有一
些特殊结构螺旋通道的流动特性被学者研究, $\mathrm{Li}$ 等 人 ${ }^{[24]}$ 就提出了如图2所示的起皱螺纹螺旋通道, 并对 其流体流动特性进行了探究, 结果发现, 其流动阻力 比光管增加了 50\% 300\%. 这些结构的提出丰富了螺 旋通道研究的范围, 为新型螺旋通道的研发提供了 参考.

\section{3 层流状态下的传热特性}

改变螺旋通道的几何参数及内部结构之所以会 对其传热性能产生影响, 是因为曲率、扭率、螺距及 其倾斜角度等参数数值的变化均会使流体受到的离 心力及浮力等发生改变, 从而使其产生的涡及二次 流的速度和分布情况发生变化. 这就意味着流体的 扰动及对边界层的减薄程度发生了变化, 传热性能 也就随之改变.

螺旋通道的努塞尔数是表征其对流传热效果的 一个无量纲参数, 与其进、出口温度及壁温有一定关 系，而进、出口温度及壁温又和流体在通道内的扰动 有关，也就是二次流的分布情况，在之前的研究中已 经指明二次流的分布和螺旋半径及螺距有关. 所以, 螺旋通道结构对其传热特性影响的研究主要集中在 螺旋管内径、螺旋半径、螺距以及截面形状上.

(i ) 实验研究. 由上述分析可知, 螺旋管内 径、螺旋半径及螺距的改变会对其传热性能产生影 响. Moawed ${ }^{[25]}$ 通过实验探究了如图1所示螺旋通道 的 $R / D$ 在7.086 16.142及 $P / D$ 在1.81 3.205范围内的传 热特性, 雷诺数和 $P / D$ 一定时, 努塞尔数随着 $R / D$ 的 增加而增大; 雷诺数和 $R / D$ 一定时, 努塞尔数随着 $P / D$ 的增加而增大. 随后, Heo等人 ${ }^{[26]}$ 通过实验研究 了螺旋通道不同几何参数对其传热特性的影响, 结

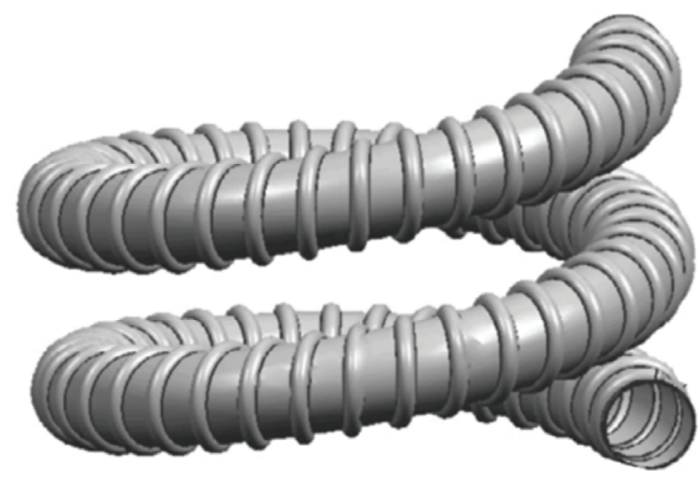

图 2 螺旋起皱的螺旋通道 ${ }^{[2]}$

Figure 2 Helical tube cooperating with helical corrugation ${ }^{[24]}$ 
果发现, 螺旋管内径增大时努塞尔数减小; 管道长度 对努塞尔数影响不大.

另外, 一些特殊的结构参数也被很多学者进行 了探究. Park等人 ${ }^{[27]}$ 发现自然对流状态下, 当螺旋通 道的倾斜角为 $80^{\circ}$ 时, 传热系数最大, 说明此时螺旋 管道内的二次流动最大程度改善了流体对边界层的 减薄情况, 扰动强度达到最大. Poskas等人 ${ }^{[28]}$ 研究了 双壁面加热矩形螺旋通道内空气流的传热特性, 结 果发现, 双壁面加热时的努塞尔数比单壁面加热时 提高 $20 \%$; 层流状态下螺旋通道的平均传热较普通 直管提高了 $50 \%$.

(ii) 模拟研究. 数值模拟的结果也在一定程度 上验证了理论分析及实验结论. Mohammed等人 ${ }^{[16]}$ 通 过数值模拟发现, 降低螺旋半径、增加螺旋管道内径 及减小螺旋管道环直径均可以增大努塞尔数; 改变 螺距对传热性能影响不大. 也有学者研究了一些其 他结构的螺旋通道, 并且从熵产的角度进行分析, Kurnia等人 ${ }^{[29]}$ 模拟了层流状态下, 不同截面形状对 螺旋通道传热及熵产的影响, 结果发现, 螺旋通道具 有比直管更高的传热系数, 并且产生的熵产更少; 正 方形的熵产最多, 椭圆次之, 而圆形最少; 冷却时产 生的熵产高于加热时的熵产; 传热熵产比黏性耗散 引起的熵产高出 2 个数量级.

\section{4 湍流状态下的传热特性}

( i ) 实验研究. 为了探究湍流及层流状态下不 同结构螺旋通道传热特性的差别, Poskas等人 ${ }^{[28]}$ 研究 了双壁面加热矩形螺旋通道内空气流的传热特性, 结果发现, 在层流及湍流状态下, 螺旋通道较普通直 管而言, 平均传热系数分别提高 $50 \%$ 和 $20 \%$; 增加螺 旋通道曲率对于流体扰动效果的提升不明显. 湍流 状态下其传热系数之所以可以提高更多, 是因为湍 流状态时, 流体的扰动已经相当剧烈, 双壁面加热对 于增强流体扰动效果没有层流状态时明显. Simonis 等人 ${ }^{[30]}$ 实验探究了矩形截面螺旋通道中表面粗糙度 对其传热特性的影响, 结果表明, 湍流状态下, 粗粘 凸表面螺旋通道的传热性能是光滑凸表面的两倍多, 并且达到了凹表面传热的水平; 粗糙凹表面和光滑 凹表面的传热性能相近, 相比于光滑直管, 其传热效 果提升 $50 \%$ 左右; 而在所研究的雷诺数范围内, 粗粘 凹、凸表面螺旋通道的传热效率均高于光管.

(ii) 模拟研究. 在湍流状态下螺旋通道传热特
性的模拟研究中, 很多学者也证实了增加扰动对其 传热特性的提高并无太大作用. 陈瑞杰等人 ${ }^{[22]}$ 模拟 探究了湍流状态下肋片对螺旋通道传热特性的强化 作用, 结果表明, 单一矩形截面螺旋通道换热壁面中 心线附近的二次流较弱, 换热效果差, 安装肋片之 后, 会在矩形截面中心处产生附加二次流, 其换热系 数提高到光管的 1.03 1.2 倍, 阻力系数为光管的 1.003 1.033倍, 强化传热因子则为光管的 0.911 1.067 倍; 并且, 低雷诺数时, 其传热效果较好. 但 是, $\mathrm{Li}$ 等人 ${ }^{[24]}$ 发现起皱螺纹传热性能比光管增加 50\% 80\%, 并随着螺纹间距的减小而增大, 说明起 皱的扰动效果要优于肋片, 可能是因为起皱部分产 生了新的二次流动, 并且新产生的二次流动对流体 的扰动效果明显优于肋片.

\section{2 流动工质}

\section{1 纳米流体}

由于纳米颗粒的加人, 纳米流体的性质和传统 单相流体有很大不同, 可以近似看作固液两相混合 的纳米流体与单相流体相比, 具有以下优点: (1) 纳 米颗粒在基液中做布朗运动, 使得纳米流体具有较 高的分散稳定性; (2) 与传统的泥浆沉积不同, 纳米 颗粒不容易形成阻塞, 可以应用到小型甚至微型设 备中; (3) 与传统工质相比, 通过提高流体扰动来增 加等效的传热效果时, 纳米流体所需的百功较少; (4) 可以通过改变纳米流体的体积分数来调整其导热系 数以适应不同应用的需求; (5) 较高的比表面积以及 固体颗粒和流体之间更多的热量传递 ${ }^{[31]}$. 这些特点 使得纳米流体得到了广泛的应用, 尤其是在强化传 热技术领域, 越来越多的研究者开始探究纳米流体 对于螺旋通道传热特性的影响.

(i) 纳米流体的传热特性. 纳米流体对螺旋通 道传热性能的提高不仅与布朗运动、布朗分散及流固 界面的摩擦有关, 分散性、流固表面的边界层及纳米 流体本身的热物理性质也在一定程度上影响着螺旋 通道传热特性. 相关的实验结果表明, 纳米流体的传 热性能不仅与纳米颗粒的加人有关, 加人纳米颗粒 之后工质自身性质的改变也是重要影响因素. 纳米 流体的热物理性能可以通过两相混合的经典公式进 行计算, 例如, 纳米流体的有效密度 ${ }^{[32]}$ 和比热容 ${ }^{[33]}$ 的计算公式分别为 


$$
\begin{gathered}
\rho_{\mathrm{NF}}=(1-\varphi) \rho_{\mathrm{BF}}+\varphi \rho_{\mathrm{NP}}, \\
c_{\mathrm{pNF}}=\frac{(1-\varphi)\left(\rho c_{\mathrm{p}}\right)_{\mathrm{BF}}+\varphi\left(\rho c_{\mathrm{p}}\right)_{\mathrm{NP}},}{(1-\varphi) \rho_{\mathrm{BF}}+\varphi \rho_{\mathrm{NP}}}
\end{gathered}
$$

由式(3)和(4)可知, 纳米颗粒的体积分数在一定程度 上决定着纳米流体的一些物理性质, 如表 1 所 示 $^{[34 \sim 44]}$, 一些学者就探究了添加纳米颗粒之后的工 质对螺旋通道传热特性的影响. 结果发现, 添加纳米 颗粒而制得的纳米流体的传热性能要优于基液; 随 着纳米颗粒体积分数的增加, 其传热效果明显提高; 层流状态下, 加人纳米颗粒对于工质传热性能的改 善要优于湍流状态; 分散有不同纳米颗粒的纳米流 体的传热性能之间存在差异. 分析其原因, 可能是纳 米颗粒自身的热物理性与基液差别很大，良好的热 物理性质使其传热效果提升明显; 纳米颗粒的小尺
寸效应，导致其具有更大的比表面积; 纳米颗粒在布 朗运动过程中可以传递更多的热量, 改善了传热效 果; 添加的纳米颗粒越多, 单位体积内的纳米颗粒数 量也就越多，其传递热量的效率就越高；层流状态 下, 流体扰动相对于湍流状态而言, 比较平缓, 加人 的纳米颗粒在流体中的宏观运动也就没有湍流状态 下的剧烈，也就导致了其对传热效果的提升效果没 有湍流状态下明显. Sivasankaran等人 ${ }^{[45]}$ 数值模拟了 层流状态下内置多孔介质的螺旋细通道内纳米流体 的传热特性, 结果表明, 增强混合程度可以提高努塞 尔数, 但是在高雷诺数下, 作用不大. 并且每种纳米 颗粒自身的物理性质不同，可能就导致了不同纳米 流体传热性能的差异. 这些分析的结论中，还有很多 需要研究者对其进行进一步的探究.

(ii ) 纳米流体的流动特性. 由于工质自身的物

\section{表 1 纳米流体对螺旋通道流动及传热特性的影响}

\begin{tabular}{|c|c|c|c|c|c|c|}
\hline 作者 & 流动状态 & 结构参数 $(\mathrm{mm})$ & 纳米颗粒 & 基液 & 体积分数(\%) & 流动及传热特性分析 \\
\hline Jamshidi等人 ${ }^{[34]}$ & 层流 & $\begin{array}{l}D=12 \\
P=21.4,26.7,30 \\
R=35,45,55\end{array}$ & $\mathrm{Al}_{2} \mathrm{O}_{3}$ & 水 & 1,2 & $\begin{array}{l}\text { 两种体积分数的纳米流体对应的流动阻力系数比 } \\
\text { 基液分别提 } 1.45 \%, 4.28 \% \text {; 努塞尔数比基液分 } \\
\text { 别提高 } 39 \%, 46 \%\end{array}$ \\
\hline $\begin{array}{l}\text { Akhavan-Behabadi } \\
\text { 等人 } \\
{[35]}\end{array}$ & 层流 & $\begin{array}{l}D=15.6 \\
P=25 \sim 95 \\
R=110,130,160\end{array}$ & 碳纳米管 & 传热油 & $0.1,0.2,0.4$ & 最大平均传热系数比基液增加 $80 \%$ \\
\hline Hashemi等人 ${ }^{[36]}$ & 层流 & $\begin{array}{l}D=14.37 \\
P=55 \\
R=162\end{array}$ & $\mathrm{CuO}$ & 传热油 & $0.5,1.0,2.0$ & $\begin{array}{l}\text { 最大压降值比基液增加 } 20.3 \% \text {; 最大平均传热系 } \\
\text { 数比基液增加 } 30.4 \%\end{array}$ \\
\hline Srinivas等人 ${ }^{[37]}$ & $\begin{array}{l}\text { 层流 } \\
\text { 湍流 }\end{array}$ & $\begin{array}{l}D=9.82 \\
P=32.5 \\
R=95\end{array}$ & $\mathrm{Al}_{2} \mathrm{O}_{3}$ & 水 & $\begin{array}{l}0.15,0.30,0.45, \\
0.60,0.75\end{array}$ & 比基液最多节能 $10.65 \%$ \\
\hline Khairul等人 ${ }^{[38]}$ & 湍流 & $\begin{array}{l}D=9 \\
P=18 \\
R=58\end{array}$ & $\begin{array}{l}\mathrm{CuO} \\
\mathrm{ZnO} \\
\mathrm{Al}_{2} \mathrm{O}_{3}\end{array}$ & 水 & $1,2,3,4$ & $\begin{array}{l}\mathrm{CuO} \text { 纳米颗粒的平均传热系数比另外两种平均提 } \\
\text { 高 } 7.14 \%\end{array}$ \\
\hline $\mathrm{Aly}^{[39]}$ & 湍流 & $\begin{array}{l}D=14.07 \\
P=31.74 \\
R=90,120,150\end{array}$ & $\mathrm{Al}_{2} \mathrm{O}_{3}$ & 水 & $0.5,1.0,2.0$ & $\begin{array}{l}\text { 流动阻力系数随着螺旋通道曲率的增加而增大, } \\
\text { 在纳米流体体积分数小于 } 2 \% \text { 范围内几乎不变; } \\
R e \text { 为 } 20634 \text { 及 } 176240 \text { 时, 体积分数 } 2.0 \% \text { 的纳米 } \\
\text { 流体的平均传热系数比基液提高 } 27 \% \text { 和 } 30 \%\end{array}$ \\
\hline Rakhsha等人 ${ }^{[40]}$ & 湍流 & $\begin{array}{l}D=8 \\
P=20 \sim 42 \\
R=42.5 \sim 100\end{array}$ & $\mathrm{CuO}$ & 水 & 0.1 & $\begin{array}{l}\text { 不同几何参数及雷诺数下压降提高14\% 16\%; 传 } \\
\text { 热效果比基液增强16\% 17\% }\end{array}$ \\
\hline $\begin{array}{l}\text { Mirfendereski等 } \\
\text { 人 }^{[41]}\end{array}$ & 层流 & $\begin{array}{l}P=45,55,72 \\
R=77.5 \sim 137.5\end{array}$ & $\mathrm{Ag}$ & 水 & 0.03 & $\begin{array}{l}\text { 压降随曲率的增加而增大, 并且不同几何参数下 } \\
\text { 其提升范围为 } 13 \% \text { 24\%; 传热效果比基液提高 } \\
3.5 \% \text { 3.8\% }\end{array}$ \\
\hline Srinivas等人 ${ }^{[42]}$ & $\begin{array}{l}\text { 层流 } \\
\text { 湍流 }\end{array}$ & $\begin{array}{l}D=9.82 \\
P=32.5 \\
R=95\end{array}$ & $\begin{array}{l}\mathrm{CuO} \\
\mathrm{TiO}_{2} \\
\mathrm{Al}_{2} \mathrm{O}_{3}\end{array}$ & 水 & $\begin{array}{l}0.3,0.6,1.0 \\
1.5,2.0\end{array}$ & $\begin{array}{l}\text { 3种纳米流体的表面强化传热效果比基液最多分 } \\
\text { 别增强 } 30.37 \%, 26.8 \%, 32.7 \%\end{array}$ \\
\hline $\begin{array}{l}\text { Bahrehmand等 } \\
\text { 人 }^{[43]}\end{array}$ & 湍流 & $\begin{array}{l}D=7,10,13 \\
P=20 \\
R=50,60,70\end{array}$ & $\mathrm{Al}_{2} \mathrm{O}_{3}$ & 水 & $0.1,0.2,0.3,0.4$ & $\begin{array}{c}\text { 体积分数分别 } 0.2 \%, 0.3 \% \text { 的纳米流体的传热系数 } \\
\text { 比基液分别提高 } 14 \%, 18 \%\end{array}$ \\
\hline Huminic等人 ${ }^{[44]}$ & 层流 & $\begin{array}{l}D=28 \\
P=115 \\
R=400\end{array}$ & $\begin{array}{l}\mathrm{CuO} \\
\mathrm{TiO}_{2}\end{array}$ & 水 & $0 \sim 2.0$ & $\begin{array}{l}\text { 体积分数 } 2.0 \% \text { 的 } \mathrm{CuO}, \mathrm{TiO}_{2} \text { 纳米流体的热交换效 } \\
\text { 率比基液分别提高 } 91 \%, 80 \%\end{array}$ \\
\hline
\end{tabular}

Table 1 Effect of nanofluid on the flow and heat transfer characteristics of helical channel 
理性质不同, 黏度、密度及比热容等参数均有差异, 从而对流体的流动特性有所影响. 以计算螺旋通道 中阻力系数为例, 公式如下:

$$
f=\frac{2 d_{\mathrm{h}} \Delta p}{\rho L u^{2}} .
$$

在几何参数及流速一致的情况下, 式(5)中流体 的密度 $\rho$ 和沿程压降 $\Delta p$ 共同决定了阻力系数的大小. 而又由式(3)可知, 纳米流体的密度是随着基液密度、 纳米颗粒密度及纳米颗粒体积分数共同决定的. 所 以, 研究者探究了纳米流体对螺旋通道流动特性的 影响. Rakhsha等人 ${ }^{[40]}$ 通过实验对湍流状态下螺旋通 道内纳米流体流动特性进行了分析, 结果表明, 相比 于以水为工质的螺旋通道, 加人纳米流体后其压降 增加了 $14 \%$ 16\%, 显然, 阻力系数也随着提高. 随 后, Khoshvaght-Aliabadi等人 ${ }^{[46]}$ 实验探究了螺旋微通 道内 $\mathrm{Cu}$-水纳米流体流动特性, 结果表明, 加人纳米 颗粒之后, 压降略有增加; 螺旋管直径较小时, 纳米 颗粒对其流动特性的影响较大.

也有一些学者通过数值模拟对其进行了研究, 其结果在一定程度上与实验结论保持一致. Narrein等 人 ${ }^{[47]}$ 选取了 $\mathrm{Al}_{2} \mathrm{O}_{3}, \mathrm{SiO}_{2}, \mathrm{CuO}$ 及 $\mathrm{ZnO} 4$ 种纳米颗粒, 颗粒浓度在 $1 \%$ 4\%之间, 粒径范围为 $25 \sim 80 \mathrm{~nm}$, 使 用的基液分别为水、乙二醇及机油, 结果发现, 纳米 流体对螺旋通道内流体的压降有小幅增加.

但是, 一些研究者却发现了实验值与模拟值之 间存在差异. Rakhsha等人 ${ }^{[40]}$ 就发现相比于以水为工 质的螺旋通道, 加人纳米颗粒后其压降的模拟值增 加了 6\% 7\%, 而实验值提高了 $14 \%$ 16\%. 分析原因 可能是由于在模拟过程中, 假定了纳米流体为均一 稳定的流体, 而实际上纳米流体为固液混合的两相 流体, 纳米颗粒在流体中存在小尺寸效应和布朗运 动, 这就造成了实验和模拟值的偏差.

\section{2 其他种类工质的流动及传热特性}

(i) 流动特性. 对于一些常规的制冷剂而言, 研究的重点主要集中在与普通直管的对比上. Gupta 等人 ${ }^{[48]}$ 对水平螺旋通道内 R-134a冷却剂的流动特性 进行了研究, 结果表明, 压降随着蒸汽平均质量流速 的增加而增大, 随饱和温度的增加而减小; 与直管相 比, 二次流使得扰动增强, 从而提高了压降. Mozafari等人 ${ }^{[49]}$ 通过实验研究了制冷剂R-600a在倾 斜螺旋通道内的压降, 结果表明, 水平螺旋通道的压
降最大; 与水平直管相比，其压降增加 $33 \%$ 157\%.

(ii) 传热特性. 基于纳米流体对螺旋通道传热 性能的改善作用, 越来越多研究者对螺旋通道内其 他工质的传热特性进行了探究. Gupta等人 ${ }^{[48]}$ 对水平 螺旋通道内 R-134a冷却剂的传热特性进行了研究, 结果表明, 平均传热系数随着蒸汽平均质量流速增 加而增大, 随饱和温度的增加而减小; 与直管相比, 二次流使得湍流增强, 从而提高了传热系数; 低质量 流量时, 螺旋通道十分适合替换直管. 随后, Mozafari 等人 ${ }^{[49]}$ 研究了 R-600a在倾斜螺旋通道内的传热, 螺 旋通道中心轴线与水平线夹角为 $30^{\circ}$ 和 $90^{\circ}$ 时其传热 系数分别达到最小值和最大值; 螺旋通道相比于直 管，其平均传热系数增加 $24 \%$ 165\%; 螺旋通道水平 放置时的综合性能评价因数比垂直时高 $15 \%$ 41\%, 说明使用螺旋通道可以改善制冷效果, 并且坚直位 置时, 其改善效果最佳.

当然, 也不乏关于气体工质的相关研究, $\mathrm{Xu}$ 等 人 ${ }^{[50]}$ 研究了临界压降条件下, 螺旋通道内 $\mathrm{CO}_{2}$ 对流 传热特性, 结果表明, 湍流状态下, 在浮力和离心力 的共同作用下, 横截面上的流动及传热分布发生改 变, 较大的轴向速度及传热系数都出现在螺旋通道 的底层, 在通道内顶部出现温度最大值. Zhang等 人 $^{[51]}$ 研究了超临界压力下 $\mathrm{CO}_{2}$ 在螺旋通道内垂直向 上流动时的混合对流换热性能, 结果表明, 在离心力 和浮力的共同作用下, 最低壁温出现在螺旋通道的 底部与外部之间; 证明了 3 个传热增强机理(由离心 力产生的二次浴、由浮力产生的二次浴和临界温度下 的大比热容)和两个传热减弱机理(浮力引起的密度 差减小使得二次流减弱及液气转化引起的传热系数 减小); 在传热减弱区域, 努塞尔数的突增是由热导 率的减小引起的; 气体温度低于临界温度时, 增加质 量流量可以增强传热效果, 而增加热通量则会减弱.

熔盐以其大比热容、低蒸汽压、低腐蚀性及化学 稳定性等优点, 常常被作为理想的换热工质. Xiao等 人 ${ }^{[52]}$ 实验探究了螺旋环形通道内熔盐流(包括 $53 \%$ 的 $\mathrm{KNO}_{3} 、 40 \%$ 的 $\mathrm{NaNO}_{2}$ 及 $7 \%$ 的 $\mathrm{NaNO}_{3}$ ) 的传热特性, 结 果表明, 环形螺旋通道的熔盐流动摩擦系数比螺旋 通道和直管的都大；熔盐温度较低且进出口直径较 小时其努塞尔数较大.

\section{3 结语与展望}

结合现有的实验和数值模拟研究, 螺旋通道可 
以产生垂直于轴向流动方向的二次流动，一些学者 通过改变螺旋通道结构, 进一步改善了流体对边界 层的减薄程度，提高传热性能; 不同种类流动工质自 身的热物理性质决定了其传热效率，近年来研究的 工质对螺旋通道的换热性能都有很大提升，尤其是 纳米颗粒的小尺寸效应，使其在基液中作无规则的 布朗运动，增加了导热系数; 在改变螺旋通道结构的 基础上, 分析不同工质对其传热特性的影响，对其结 构的优化和工质的选取有很大帮助.

但是，在这个领域仍有很多问题需要研究者对 其进行更加深人的探究. 结构方面，首先，现有关于 螺旋通道流动和传热特性的研究大多数集中在传统 尺度，而微尺度螺旋通道以其结构和性能上的优势 越来越受研究者的欢迎. 所以，可以从微尺度的角度 出发, 探究微细螺旋通道内单相及两相流体的流动 及传热特性, 特别是微尺度与传统尺度下流动和传 热特性产生差异的机理研究, 把传统尺度螺旋通道 的强化传热技术，应用到微尺度领域进行研究，建立 完整的微细螺旋通道强化传热体系，以便更好地满 足工程实际的换热需求; 其次, 可以从热力学第二定 理出发，探究不可逆过程中的熵增问题，以及提升相
同传热效果时所消耗的不可逆功及泵功，以便对不 同结构的螺旋通道进行统一的功耗评价，从而篮选 出节能高效的换热方案; 最后, 可以对几何参数优化 后的螺旋通道以及螺旋微细通道增加合理的扰流装 置, 并分析其流动及传热特性, 并且探究添加纳米颗 粒的工质对其性能的影响.工质方面，首先应该考虑 其稳定性，在外界物理条件(如温度、压力、磁场、 电场等)的影响下，确保工质可以长时间保持稳定, 尤其是纳米流体的稳定性缺乏统一的标准，制备纳 米流体的技术不尽相同，很难就不同研究者关于同 一纳米流体的稳定性进行比较, 这样就造成了纳米 流体在螺旋通道中的研究缺乏统一的参考，所以，很 有必要建立一个统一的纳米流体工质物理性质评价 标准, 以此来保证研究者所使用的纳米流体工质具 有相互对比的资格; 其次, 在研究纳米流体对螺旋通 道传热性能提高的同时，应该分析其压降及黏度的 增加, 并且对由于其压降及黍度增加带来的流动阻 力、不可逆功损失及泵功进行定量分析, 从而保证螺 旋通道传热性能的提升不是以更大的流动阻力为前 提的, 并且建立一个综合评价纳米流体及其他工质 对螺旋通道整体性能影响的新标准.

\section{参考文献}

1 Goering D J, Humphrey J A C, Greif R. The dual influence of curvature and buoyancy in fully developed tube flows. Heat Mass Transfer, 1997, 40: 2187-2199

2 Wu S Y, Chen Y, Li Y R, et al. Exergy transfer evaluation on enhanced heat transfer performance through tube with constant heat flux (in Chinese). J Therm Sci Tech, 2006, 2: 133-138 [吴双应，陈燕，李友荣，等. 恒热流工况下强化传热管传热能评价. 热科学与技术, 2006, 2: 133-138]

3 Ko T H. Thermodynamic analysis of optimal curvature ratio for fully developed laminar forced convection in a helical coiled tube with uniform heat flux. Int J Therm Sci, 2006, 45: 729-737

4 Ko T H, Ting K. Optimal Reynolds number for the fully developed laminar forced convection in a helical coiled tube. Energy, 2006, 31: $2142-2152$

5 Cioncolini A, Santini L. On the laminar to turbulent flow transition in diabatic helically coiled pipe flow. Exp Therm Fluid Sci, 2006, 30: 653-661

6 Chen Y, Chen H J, Zhang B Z, et al. Fluid flow and convective heat transfer in a rotating helical square duct. Int J Therm Sci, 2006, 45: $1008-1020$

7 Sundar L S, Singh M K. Convective heat transfer and friction factor correlations of nanofluid in a tube and with inserts: A review. Energy Rev, 2013, 20: 23-35

8 Li Y J, Zhou J E, Tung S, et al. A review on development of nanofluid preparation and characterization. Powder Tech, 2009, 196: 89-101

9 Aghabozorg M H, Rashidi A, Mohammadi S. Experimental investigation of heat transfer enhancement of $\mathrm{Fe}_{2} \mathrm{O}_{3}-\mathrm{CNT}_{\text {water }}$ magnetic nanofluids under laminar, transient and turbulent flow inside a horizontal shell and tube heat exchanger. Exp Therm Fluid Sci, 2016, 72: $182-189$

10 Fsadni A M, Whitty J P M, Stables M A. A brief review on frictional pressure drop reduction studies for laminar and turbulent flow in helically coiled tubes. Appl Therm Eng, 2016, 109: 334-343

11 Ghobadi M, Muzychka Y S. Pressure drop in mini-scale coiled tubing. Exp Therm Fluid Sci, 2014, 57: 57-64 
12 Zhang L, Xing Y W, Wu J H. Flow characteristics in helical duct with rectangular cross section (in Chinese). J Chem Ind Eng, 2010, 5: 1089-1096 [张丽, 邢彦伟, 吴剑华. 矩形截面螺旋通流体的流动特性. 化工学报, 2010, 5: 1089-1096]

13 Ma Y, Cao W J, Lin M. Secondary flow characteristics in helical rectangular channel (in Chinese). J Xi'an Jiaotong Univ, 2014, 8: 122-127 [马源, 曹文瑾, 林梅. 矩形截面螺旋通道内二次流流场特性. 西安交通大学学报, 2014, 8: 122-127]

14 Liu X F, Xia G D, Yang G. Experimental study on the characteristics of air-water two-phase flow in vertical helical rectangular channel. Int J Multiphase Flow, 2015, 73: 227-237

15 Liu X F, Xia G D, Yang G. Flow characteristics of slug flow in helical rectangular channel (in Chinese). J Chem Ind Eng, 2014, 65: 4231-4237 [刘献飞, 夏国栋, 杨光. 矩形截面螺旋通道内弹状流的流动特性. 化工学报, 2014, 65: 4231-4237]

16 Mohammed H A, Narrein K. Thermal and hydraulic characteristics of nanofluid flow in a helically coiled tube heat exchanger. Int Commun Heat Mass Transf, 2012, 39: 1375-1383

17 Wu S Y, Chen S J, Li Y R, et al. Numerical simulation of thermal-dynamic characteristics through a helical coiled tube with annular cross section for laminar flow (in Chinese). Nucl Power Eng, 2009, 30: 86-90 [吴双应，陈素君，李友荣，等. 环形截面螺旋通道内层流换热 热力性能数值模拟. 核动力工程, 2009, 30: 86-90]

18 Nobari M R H, Shiniyan B, Mirzaei M. Mixed convection in a vertical helical annular pipe. Int J Heat Mass Transf, 2014, 73: 468-482

19 Zhang L, Li J Q, Zhang C M, et al. Fluid flow in rectangular helical channels with vortex generator (in Chinese). J Chem Ind Eng, 2014, 65: 3838-3845 [张丽, 李佳其, 张春梅, 等. 安装浴发生器的矩形截面螺旋通内流体流动. 化工学报, 2014, 65: 3838-3845]

20 Saffari H, Moosavi R, Gholami E, et al. The effect of bubble on pressure drop reduction in helical coil. Exp Therm Fluid Sci, 2013, 51: 251-256

21 Xia G D, Liu X F. An investigation of two-phase flow pressure drop in helical rectangular channel. Int Commun Heat Mass Transf, 2014, 54: $33-41$

22 Chen R J, Ma Z Y, Wang X D, et al. Heat transfer enhancement of ribs in a helical duct with rectangular cross section (in Chinese). J Shenyang Univ Chem Tech, 2014, 28: 248-252 [陈瑞杰, 马中元, 王兴斗, 等. 肋片强化矩形截面螺旋通道换热特性研究. 沈阳化工 大学学报, 2014, 28: 248-252]

23 Li Y X, Zhang T, Zhang C M, et al. Enhanced heat transfer mechanism of winglet vortex generator in helical channel with semicircular cross section (in Chinese). J Chem Ind Eng, 2016, 67: 1814-1821 [李雅侠, 张腾, 张春梅, 等. 翼型浴发生器对半圆形螺旋通道的换 热强化机理. 化工学报, 2016, 67: 1814-1821]

$24 \mathrm{Li} \mathrm{Y} \mathrm{X,} \mathrm{Wu} \mathrm{J} \mathrm{H,} \mathrm{Wang} \mathrm{H,} \mathrm{et} \mathrm{al.} \mathrm{Fluid} \mathrm{flow} \mathrm{and} \mathrm{heat} \mathrm{transfer} \mathrm{characteristics} \mathrm{in} \mathrm{helical} \mathrm{tubes} \mathrm{cooperating} \mathrm{with} \mathrm{spiral} \mathrm{corrugation.} \mathrm{Energy}$ Procedia, 2012, 17: 791-800

25 Moawed M. Experimental study of forced convection from helical coiled tubes with different parameters. Energy Convers Manage, 2011, 52: 1150-1156

26 Heo J H, Chung B J. Influence of helical tube dimensions on open channel natural convection heat transfer. Int J Heat Mass Transf, 2012, 55: 2829-2834

27 Park J H, Moon J Y, Chung B J. Natural convection of an inclined helical coil in a duct. Int Commun Heat Mass Transf, 2014, 59: 11-16

28 Poskas P, Simonis V, Ragaisis V. Heat transfer in helical channels with two-sided heating in gas flow. Int J Heat Mass Transf, 2011, 54: $847-853$

29 Kurnia J C, Sasmito A P, Shamim T, et al. Numerical investigation of heat transfer and entropy generation of laminar flow in helical tubes with various cross sections. Appl Therm Eng, 2016, 102: 849-860

30 Simonis V, Poskas P, Ragaisis V. Enhancement of heat transfer and hydraulic drag in gas-cooled helical channels with artificial roughness on convex wall. Nuclear Eng Design, 2012, 245: 153-160

31 Wen D, Lin G, Vafaei S, et al. Review of nanofluids for heat transfer applications. Particuology, 2009, 7: 141-150

32 Xuan Y, Roetzel W. Conceptions for heat transfer correlation of nanofluids. Int J Heat Mass Transf, 2000, 43: 3701-3707

33 Pak B C, Cho Y I. Hydrodynamic and heat transfer study of dispersed fluids with submicron metallic oxideparticles. Exp Heat Transf, 1998, 11: 151-170

34 Jamshidi N, Farhadi M, Sedighi K, et al. Optimization of design parameters for nanofluids flowing inside helical coils. Int Commun Heat Mass Transf, 2012, 39: 311-317

35 Akhavan-Behabadi M A, Pakdaman M F, Ghazvini M. Experimental investigation on the convective heat transfer of nanofluid flow inside vertical helically coiled tubes under uniform wall temperature condition. Int Commun Heat Mass Transf, 2012, 39: 556-564

36 Hashemi S M, Akhavan-Behabadi M A. An empirical study on heat transfer and pressure drop characteristics of CuO-base oil nanofluid flow in a horizontal helically coiled tube under constant heat flux. Int Commun Heat Mass Transf, 2012, 39: 144-151

37 Srinivas T, Vinod A V. Performance of an agitated helical coil heat exchanger using $\mathrm{Al}_{2} \mathrm{O}_{3} /$ water nanofluid. Exp Therm Fluid Sci, 2013, 51: $77-83$ 
38 Khairul M A, Saidur R, Rahman M M, et al. Heat transfer and thermodynamic analyses of a helically coiled heat exchanger using different types of nanofluids. Int J Heat Mass Transf, 2013, 67: 398-403

39 Aly W I A. Numerical study on turbulent heat transfer and pressure drop of nanofluid in coiled tube-in-tube heat exchangers. Energy Convers Manage, 2014, 79: 304-316

40 Rakhsha M, Akbaridoust F, Abbassi A, et al. Experimental and numerical investigations of turbulent forced convection flow of nano-fluid in helical coiled tubes at constant surface temperature. Powder Tech, 2015, 283: 178-189

41 Mirfendereski S, Abbassi A, Saffar-Avval M. Experimental and numerical investigation of nanofluid heat transfer in helically coiled tubes at constant wall heat flux. Adv Powder Tech, 2015, 26: 1483-1494

42 Srinivas T, Vinod A V. Heat transfer intensification in a shell and helical coil heat exchanger using water-based nanofluids. Chem Eng Proc-Process Int, 2016, 102: 1-8

43 Bahrehmand S, Abbassi A. Heat transfer and performance analysis of nanofluid flow in helically coiled tube heat exchangers. Chem Eng Res Design, 2016, 109: 628-637

44 Huminic G, Huminic A. Heat transfer and entropy generation analyses of nanofluids in helically coiled tube-in-tube heat exchangers. Int Commun Heat Mass Transf, 2016, 71: 118-125

45 Sivasankaran S, Narrein K. Numerical investigation of two-phase laminar pulsating nanofluid flow in helical microchannel filled with a porous medium. Int Commun Heat Mass Transf, 2016, 75: 86-91

46 Khoshvaght-Aliabadi M, Pazdar S, Sartipzadeh O. Experimental investigation of water based nanofluid containing copper nanoparticles across helical microtubes. Int Commun Heat Mass Transf, 2016, 70: 84-92

47 Narrein K, Mohammed H A. Influence of nanofluids and rotation on helically coiled tube heat exchanger performance. Thermochim Acta, 2013, 564: 13-23

48 Gupta A, Kumar R. Condensation of R-134a inside a helically coiled tube-in-shell heat exchanger. Exp Therm Fluid Sci, 2014, 54: $279-289$

49 Mozafari M, Akhavan-Behabadi M A, Qobadi-Arfaee H, et al. Condensation and pressure drop characteristics of R600a in a helical tube-in-tube heat exchanger at different inclination angles. Appl Therm Eng, 2015, 90: 571-578

$50 \mathrm{Xu} \mathrm{J}$, Yang C, Zhang W, et al. Turbulent convective heat transfer of $\mathrm{CO}_{2}$ in a helical tube at near-critical pressure. Int J Heat Mass Transf, 2015, 80: 748-758

51 Zhang W, Wang S, Li C, et al. Mixed convective heat transfer of $\mathrm{CO}_{2}$ at supercritical pressures flowing upward through a vertical helically coiled tube. Appl Therm Eng, 2015, 88: 61-70

52 Xiao P, Guo L, Zhang X. Investigations on heat transfer characteristic of molten salt flow in helical annular duct. Appl Therm Eng, 2015, 88: $22-32$ 
Summary for “螺旋通道内流体流动与传热特性研究进展”

\title{
A review on fluid flow and heat transfer in helical channel
}

\author{
LIN Qing $\mathrm{u}^{1,2^{*}}$, LIU PengHui ${ }^{1,2}$, FENG ZhenFei ${ }^{1,2} \&$ ZHU Li ${ }^{1,2}$ \\ ${ }^{1}$ Guangxi Key Laboratory of Petrochemical Resource Processing and Process Intensification Technology, Guangxi University, Nanning 530004, \\ China; \\ ${ }^{2}$ School of Chemistry and Chemical Engineering, Guangxi University, Nanning 530004, China \\ * Corresponding author, E-mail: linqy121@gxu.edu.cn
}

As an important structure to improve the efficiency of heat transfer and mass transfer, helical channel has been widely used in the application fields of power generation, refrigeration, chemical reaction, heat recovery, food processing and material mixing because of its compact structure and easy processing.

Different from the straight channel, helical channel can produce the secondary flow perpendicular to the axial flow direction, which can increase the disturbance of the fluid flowing in the channel, and then reduce the thickness of the boundary layer, and enhance the heat transfer characteristics. The flowing state of the liquid in helical channel becomes relatively complicated because of the curvature and torsion of helical channel, the centrifugal force and centripetal force cause the fluid to flow toward the wall and form the Dean vortex, and then flow field and velocity field will be changed.

In addition to the use of helical channel instead of straight channel to improve the heat transfer efficiency of the passive heat transfer technology, the use of efficient heat exchange quality can further improve the performance of heat transfer equipment. For example, the nanofluid is prepared by adding nanoparticles and dispersing agent in the base liquid, because of higher thermal conductivity of nanoparticles and the Brown movement, it is widely used in the heat exchanger.

The flow and heat transfer characteristics of the fluid in the helical channel have become a hot research topic in recent years, and they are of crucial guiding significance for practical applications. At present, the essential reason for the secondary flow is that the centrifugal force produced by the curvature and torsion of the helical channel can affect the fluid, however, with the secondary flow, the flow resistance will increase with the increase of heat transfer. On the other hand, the addition of nanoparticles in the base fluid not only has a significant improvement on the heat transfer coefficient, but also has higher flow resistance compared with the base fluid. The results show that the flow resistance increases with the increase of heat transfer characteristics under the two conditions of heat transfer enhancement. At the same time, how to reduce the increase of flow resistance is the key to optimize the heat transfer characteristics of helical channel.

The research focused on the structural parameters of helical channel and the working fluid, and the research progress of fluid flow and heat transfer in helical channel was reviewed. The influence of different structures and working fluid on the characters of fluid flow and heat transfer in helical channel were summarized. The effect of structure parameters such as coiled curvature radius, coil diameter, coil pitch, cross-section shape and physical property parameters such as their kinds and concentrations on heat transfer and flow resistance were analyzed. The conclusions of experiments and simulations in laminar and turbulent flows were compared. Reference for structure optimization and working fluid selection of helical channel were provided, and the development directions of fluid flow and heat transfer characteristics in helical channel were also proposed.

helical channel, structure, working fluid, flow characteristics, heat transfer characteristics

doi: 10.1360/N972016-01134 